\title{
CLEARY, Matthew; STOKES, Susan. 2006. Democracy and the Culture of Skepticism. Political Trust in Argentina and Mexico. New York: Russell Sage Foundation, 244 pp.
}

Ledly HENRÍQuEZ ${ }^{1}$

Es frecuente en la actualidad escuchar acerca de una cultura sustentada en el individualismo, la apatía y la falta de confianza, tanto entre ciudadanos como hacia sus representantes políticos. Existe incluso un debate que trasciende los círculos académicos y que tiende a vincular el tema de la calidad de la democracia con la mayor o menor participación de los individuos en la vida social y política. En este contexto, el título del libro puede sugerir un tratamiento de esta temática en la línea de asociar la cultura del escepticismo a una menor calidad democrática. Nada más lejos del propósito de los autores ${ }^{2}$, cuyo argumento central consiste en sostener lo contrario, es decir, que la cultura política que más aporta a la consolidación democrática no es aquella sustentada en la participación y confianza de los ciudadanos entre sí y en sus representantes políticos, sino aquella que se asienta en redes sociales más bien débiles y en una actitud de escepticismo hacia éstos.

1. Psicóloga, Pontificia Universidad Católica de Chile. Magíster (C) en Ciencia Política, Universidad de Chile. Correo electrónico: Ledly1@hotmail.com.

2. Susan Stokes es profesora de Ciencia Política en la Universidad de Yale. Matthew Cleary es profesor asistente de Ciencia Política en Maxwell School, Syracuse University. Ambos centran sus investigaciones en la teoría de la democracia en países en vías de desarrollo, siendo su foco de interés Latinoamérica. La publicación de este libro corresponde a una serie de investigaciones realizadas por la Fundación Rusell Sage acerca del rol de la confianza y el comportamiento cooperativo en el establecimiento y mantención de regímenes democráticos. El libro constituye un valioso aporte a la serie de estudios de esta fundación, al ser el único que otorga una aproximación de esta temática en el contexto latinoamericano. 
I.

La primera parte del libro busca situar el rol de la cultura política en la teoría de la democracia, circunscribiendo este concepto al lugar ocupado por la variable "confianza en los regímenes democráticos", y desde un enfoque metodológico sustentado en la teoría de juegos. Cleary y Stokes identifican y cuestionan los principales argumentos de la teoría del capital social, rebatiendo el planteamiento de que es posible observar democracias más consolidadas en países que poseen una cultura basada en la confianza recíproca entre sus ciudadanos y hacia sus representantes. Se exponen en primera instancia los principales postulados de la teoría del capital social, citando para ello a Robert Putnam y Pamela Paxton entre otros (p. 4). El capital social es definido como asociaciones entre individuos o redes sociales que se caracterizan por la existencia de reciprocidad, confianza y sentimientos positivos entre sus miembros, generando relaciones sustentadas en la cooperación. El capital social reforzaría la democracia por las siguientes razones: las asociaciones cívicas actuarían como escuelas para la vida en democracia en tanto promueven el logro de objetivos comunes, estimulan la cooperación con el gobierno en la provisión de servicios y a su vez lo liberan de la tarea de destinar tiempo y recursos a reforzar lazos de confianza con sus ciudadanos, pudiendo invertirlos en otras actividades. En resumen, esta teoría establece la siguiente relación causal: la confianza entre ciudadanos estimula una mayor participación y cooperación cívica, aspecto que influye de manera positiva en la consolidación democrática.

Sin embargo, esta relación causal es rebatida y cuestionada en el libro, al introducir las variables de "confianza personal y confianza institucional" en relación a la consolidación democrática ${ }^{3}$. El concepto de confianza es definido como una relación entre actores en torno a una acción, en donde "si A y B son actores y X es una acción en la cual A está interesado, entonces si A cree que B hará X, A confiará en B" (p. 11) ${ }^{4}$. Al respecto, los autores realizan una distinción de especial importancia para sustentar el argumento de su libro, apoyándose en el dilema del prisionero (tipo de juego no cooperativo). El argumento consiste en señalar que en muchas situaciones, A confía en que B realizará determinada acción sólo mediante la existencia de castigos directos asociados a la falta de cooperación, sanciones que pueden ser impartidas ya sea por una de las partes involucradas o por un tercer actor, y no por la creencia en las cualidades inherentes de la otra parte involucrada. De acuerdo a estos argumentos, es posible

3. Para los autores, concordando con Linz y Stepan, es posible hablar de un régimen democrático consolidado cuando la mayor parte de la opinión pública posee la creencia que los procedimientos e instituciones democráticas son los más apropiados para gobernar la vida colectiva en una sociedad (p. 32).

4. Los autores excluyen como relación de confianza la espontánea coincidencia de intereses entre A y B en torno a una acción X. 
identificar dos tipos de relaciones de confianza: personal e institucional. La primera de éstas se extiende a aquellas situaciones donde es inherente confiar en el otro, pues ambos obtendrán ganancias, siendo un tipo de juego cooperativo. Por el contrario, la confianza institucional se sustenta en un tipo de juego no cooperativo, donde los ciudadanos apoyan los mecanismos de control institucional como modo de establecer sanciones e incentivos sobre sus representantes políticos, para garantizar el cumplimiento de sus responsabilidades políticas. Dicho de otra manera, la confianza institucional se sustenta en la desconfianza que poseen los ciudadanos en que sus representantes políticos, en ausencia de restricciones, velarán adecuadamente por sus intereses. De este modo, la confianza institucional se encuentra en profunda tensión con la confianza personal depositada en los políticos, pudiendo concluirse "que la desconfianza personal, acompañada (en el mejor de los casos) por una confianza (débil) institucional, refuerza la calidad del gobierno representativo" (p. 14).

Esta afirmación es contrastada a través de un estudio comparado de Argentina y México. Se establecen criterios comparativos en tres niveles fundamentales: comparación entre países, entre regiones al interior de cada país ${ }^{5}$ y entre las regiones de ambas naciones. El estudio fue realizado en los países mencionados, pues además de situarse en los extremos geográficos de Latinoamérica, norte y sur, poseen tanto una trayectoria histórica y de democratización distinta, así como desafíos de consolidación democráticos diferentes ${ }^{6}$.

En este contexto teórico y regional, el principal aporte del libro, que puede ser calificado de original y enriquecedor, radica en el establecimiento de un continuo en torno a la variación regional de la calidad de la democracia, así como de un modelo explicativo de estas diferencias fundado en la variable geográfica. Por otro lado, debe destacarse su valor frente a estudios previos que vinculan confianza y democracia tan sólo a nivel de comparación entre países, siendo realizados además la mayoría de las veces en países industrializados.

5. Se introduce además la comparación entre municipalidades al interior de cada provincia o estado, aspecto que enriquece aún más el estudio.

6. Se realiza en el libro una buena descripción de los aspectos mencionados, de la cual es conveniente destacar que los procesos de democratización más recientes en Argentina y México son de naturaleza distinta, caracterizándose el primero por el fin del régimen militar, mientras el segundo está marcado por la pérdida del poder hegemónico del Partido Revolucionario Institucional (PRI) tras las penúltimas elecciones presidenciales. Cabe mencionar que los autores han realizado investigaciones previas en ambos países. Stokes posee una serie de publicaciones referidas a Argentina, entre las que se pueden mencionar "Clientelism and Democracy: Evidence from Argentina" (et al., 2002), "Vote Buying in Argentina" (et al., 2004) y "Perverse Accountability: a Formal Model of Machine Politics with Evidence from Argentina" (2005). Cleary, por su parte, ha realizado diversas publicaciones respecto a México, entre las cuales es posible consignar "Electoral Competition, Citizen Influence, and Government Performance in Mexican Municipalities" (2003) y "Electoral Competition and Democracy in Mexico" (2004). 
No es posible obviar la metodología utilizada en esta investigación, pues sin duda permite respaldar sus principales conclusiones, siendo precisa y consistente. Se recurre a una metodología cuantitativa y cualitativa, siendo la primera la que otorga el mayor sustento a los resultados de la investigación. Los instrumentos utilizados para realizar análisis estadísticos, que permiten establecer conclusiones sustentadas en correlaciones significativas, se encuentran disponibles en el apéndice del libro. El análisis cuantitativo se complementa por la utilización de resultados de investigaciones anteriores realizadas por otros autores en estos países ${ }^{7}$. Se recurrió además a metodologías cualitativas, en especial entrevistas, para recabar información vinculada a las creencias de una muestra de ciudadanos $^{8}$ a través de cada región, en torno a la variable "confianza personal/institucional".

En cuanto a la argumentación central, la investigación contrasta las siguientes hipótesis (p. 15):

1. En lugares donde la democracia se encuentra relativamente consolidada y funciona bien, la forma de confianza más difundida debiese ser la institucional, mientras que en el caso contrario debiese primar la confianza personal.

2. En las regiones donde prima el clientelismo, se espera encontrar una cultura política de confianza personal en los representantes políticos, en desmedro de una cultura política sustentada en la confianza institucional.

3. No debiesen existir diferencias sistemáticas en la calidad de la vida asociativa, prevalencia del capital social, al comparar regiones con mayor y menor consolidación democrática. Además, los niveles de confianza institucional no deberían lograr predecirse a partir de los niveles de confianza personal entre los ciudadanos (p. 15).

II.

En la segunda parte de este libro se identifican las regiones estudiadas, realizándose una descripción detallada de sus principales características, tanto en las áreas económica, política y social, estableciéndose además

7. Véanse a Remmer y Wibbels (2000) acerca de las diferencias regionales en el desempeño fiscal en Argentina, así como Calvo y Murillo (2004) respecto al comportamiento fiscal de los gobiernos provinciales en Argentina.

8. Se conformó una muestra en cada país para el periodo 2001-2002, lapso que coincidía con procesos electorales, tanto en México como en Argentina. En Argentina se realizaron entrevistas a 480 adultos en las provincias de Buenos Aires, Córdoba y Misiones, y a 480 adultos en la provincia de General Pueyrredón, B.A., la cual contiene la ciudad de Mar del Plata. En México se realizaron entrevistas a 400 adultos por estado (Baja California, Chihuahua, Michoacán y Puebla). Los criterios metodológicos para la selección de las respectivas muestras se detallan en el libro. 
un ranking en torno a la calidad de la democracia regional al interior de cada país. Corresponde señalar que en los acápites de esta parte no se pretende establecer un modelo explicativo de las causas de estas diferencias, sino solamente explicitar estas disparidades, apoyándose para ello en investigaciones previas ${ }^{9}$.

Respecto a México, para lograr establecer la variación de la calidad democrática a nivel regional, se identifican como variables de análisis "competencia electoral y alternación partidaria en el poder", "violencia política y violaciones a los derechos humanos", "actividad legislativa y su independencia del Estado central", y finalmente "opinión pública acerca de la democracia". Como principal conclusión, se establece que la consolidación democrática en estos cuatro estados está determinada por la competencia partidaria. Es así como los estados de Baja California y Chihuahua, en ese orden respectivamente, exhiben mayores niveles de consolidación democrática, en tanto el Partido Revolucionario Institucional (PRI) debió adaptar sus estrategias partidarias a la competencia electoral. En efecto, en Baja California, el PRI aceptó la nueva realidad electoral al consolidarse la victoria de Ernesto Ruffo, perteneciente al Partido de Acción Nacional (PAN), como gobernador en el año 1989. Si bien en Chihuahua el PRI se ha mantenido como partido dominante, salvo en las elecciones de 1983 y 1992, las acusaciones de fraude electoral y posteriores protestas tras la sorpresiva derrota del PAN en las elecciones parlamentarias de 1986, iniciaron un proceso de consolidación democrática. Éste se caracterizó por la adaptación del PRI a un sistema electoral más competitivo, adecuando sus estrategias internas ${ }^{10}$. Baja California es situada en primer lugar respecto a Chihuahua, pues este estado, aún bajo el peak de la hegemonía del PRI, mostró una realidad distinta a la de otras regiones, caracterizada por una mayor competencia electoral. Mientras en el resto de los estados el PRI obtenía entre un 85\% y un 100\% de la votación, en Baja California el PAN obtenía regularmente el $30 \%$.

A cambio, la democracia tendría un menor grado de consolidación en Michoacán y Puebla: mientras que en 1983, en los estados del norte de México (Baja California y Chihuahua), se producían importantes cambios en torno a la competencia electoral, en Michoacán el PRI dominaba las elecciones. La competencia partidaria se califica como baja en este estado, al existir prácticas asociadas al clientelismo y al caciquismo. Una clara prueba de ello es la figura política de Cuauhtémoc Cárdenas. Si

9. Con los datos disponibles, se realizan análisis multivariables, simulaciones y regresiones estadísticas, considerando distintas variables para cada país.

10. Al respecto, se señala que el PAN, luego de su triunfo en 1983, no fue capaz de consolidar la movilización política y social que acompañó su éxito, razón por la cual en 1986, al tiempo que Ruffo era electo gobernador en Baja California, el PAN perdía las elecciones en Chihuahua. Esta derrota inesperada fue un factor detonante en las protestas de 1986. 
bien éste abandonó el PRI y conformó el Partido de la Revolución Democrática (PRD), la competencia electoral no aumentó, primando las prácticas clientelares mantenidas por ambos partidos y reforzadas por el antagonismo entre sus principales figuras políticas. A esto debe adicionarse los brotes de violencia tras las elecciones gubernamentales de 1989 y 1992, al cuestionar el PRD la victoria del PRI. Por su parte, Puebla se ha caracterizado por la total hegemonía del PRI, pudiendo plantearse que hasta 1990 las elecciones no fueron competitivas. Aunque a partir de 1995 es posible detectar elecciones más competitivas a nivel municipal en Puebla, el PRI se ha mantenido como partido dominante, controlando las gobernaciones y los puestos en el Congreso. La primacía del PRI, pese a que puede ser atribuida a una oposición débil, también se explica por el control que ejerce este partido sobre el proceso electoral, recurriendo a prácticas clientelares, movilización, intimidación y fraude ( $p$. 50). De este modo, para los autores, Puebla posee una democracia menos consolidada en comparación a Michoacán.

En Argentina se establece que el más alto nivel de consolidación democrática se observa en Mar del Plata, seguido de manera decreciente por las provincias de Buenos Aires, Córdoba y Misiones. Para ello, una de las variables analizadas es el comportamiento fiscal de los gobiernos provinciales. Se establece que la sobre representación de las provincias más pequeñas en el Congreso puede constituir una fuente de relaciones clientelares y patrocinio desde el gobierno central hacia el nivel provincial y en definitiva hacia los ciudadanos que pertenecen a éstas. Este factor es precisado aún más al complementarse con un indicador que abarca el número de habitantes por provincia y su nivel de pobreza, logrando establecerse que de aquellas provincias más pequeñas, las más populosas y no las más pobres son las que reciben mayor apoyo fiscal de manera informal ${ }^{11}$. Se incluye además la variable "volatilidad del voto a nivel provincial", esperándose que en aquellas regiones más democráticas y libres de clientelismo, la intención de voto cambie de un partido a otro y también entre elecciones. Se concluye que las provincias de Buenos Aires (pese a que puede ser considerada un bastión del peronismo) y Córdoba se evidencian como más democráticas que la de Misiones ${ }^{12}$.

Finalmente se analiza el caso particular de Mar del Plata, ciudad perteneciente a la provincia de Buenos Aires, por considerarla un caso palmario de cultura institucional. Esta afirmación se sustenta en el referéndum

11. Se realizó un análisis estadístico que asocia las variables "número de habitantes en determinadas municipalidades" y "gastos en personal en el sector público", determinándose por ejemplo que las municipalidades de Misiones poseen un mayor gasto en personal (pp. 60-62).

12. Esta conclusión es extraída del análisis de los alcaldes electos en 1991 y 1995 en las provincias de Córdoba, Misiones y Buenos Aires entre otras. Si bien tanto Misiones como Buenos Aires predominaron en ambos periodos los alcaldes peronistas, la volatilidad del voto peronista fue mayor en Buenos Aires que en Misiones. 
de 1996 convocado por el alcalde radical Elio Aprile y destinado a aprobar un impuesto especial para financiar obras públicas. Si bien legalmente no era necesaria la consulta para aprobar dicho impuesto, ésta permitió legitimar ante la ciudadanía la gestión del alcalde.

\section{III.}

La tercera parte de este libro tiene como objetivo contrastar las hipótesis de cultura política y democracia planteadas en la parte I, así como constatar la validez del ranking respecto a los grados de consolidación democrática señalado en la parte II, estableciendo para ello sus principales hallazgos en torno a cinco variables: "confianza en instituciones y confianza en políticos"; "elecciones, confianza y responsabilidad"; "confianza personal y clientelismo"; "democracia y grado de apego a las normas de la ley (irrestricto/consentimiento contingente)"; y "participación cívica".

Respecto a la primera de estas variables, tanto en Argentina como en México, se concluyó que prima la confianza institucional por sobre la confianza personal. No obstante, al realizar un análisis estadístico regional, aquellos estados o provincias que mostraron mayores niveles de consolidación democrática evidenciaron mayor confianza en controles institucionales, mientras que las regiones que fueron calificadas como menos democráticas expresaban mayor confianza personal en sus representantes políticos. Si bien en ambos países primó la confianza institucional, avalando la hipótesis de la cultura política del escepticismo (los regímenes democráticos se sustentan en la desconfianza hacia sus representantes políticos), los niveles de confianza institucional fueron más altos en Argentina que en México ${ }^{13}$.

Al considerar la variable "elecciones, confianza y responsabilidad política", se buscó establecer si la muestra otorgaba su apoyo a un determinado partido por considerar que su programa era el mejor o porque éste concedía favores a sus constituyentes. En Argentina, los resultados del análisis multivariable fueron consistentes con las hipótesis planteadas, existiendo mayores niveles de confianza institucional en las regiones más democráticas, en especial Mar del Plata, aspecto que decreció de acuerdo al continuo regional establecido en las provincias menos democráticas. Si bien en México los resultados fueron consistentes con los de Argentina, la magnitud de las diferencias regionales fue menor, siendo Baja California una de las regiones con más alto rango de no respuesta en las preguntas relacionadas con esta variable.

13. Por ejemplo, un $81 \%$ de argentinos versus un $63 \%$ de mexicanos cree que los políticos ponen atención en los ciudadanos porque quieren ser reelectos y no porque estén realmente interesados en sus demandas (p. 90). 
Al contrastar la hipótesis planteada por los investigadores respecto al clientelismo y la confianza personal, los resultados estadísticos permiten concluir que el clientelismo induce confianza personal. Aunque esta conclusión puede parecer poco novedosa, el aporte principal de los autores respecto a este tópico consiste en señalar que el "clientelismo" se comportaría como variable dependiente de la variable "geográfica o regional". Este aspecto se profundizará al exponer el modelo explicativo de los investigadores, donde la relación entre estrategias partidarias y variable geográfica determina la primacía de relaciones basadas ya sea en la confianza personal o en la confianza institucional ${ }^{14}$.

Con relación a la cuarta variable identificada en esta investigación, de ser cierta la hipótesis que asocia consolidación democrática a confianza institucional, el análisis estadístico debiese mostrar un apego a las normas de la ley más alto en las regiones más democráticas en comparación a las no democráticas. Tal planteamiento es confirmado en México, donde en los estados más democráticos la muestra se mostró proclive a cumplir con las normas legales vigentes y a sancionar aquellas conductas fuera del margen de la ley, en contraposición con aquellos estados menos democráticos, donde el apego a las normas de la ley fue relativizado. Aunque en Argentina es posible plantear que se produce un continuo desde las provincias menos democráticas hasta las más democráticas en torno al apego a la ley, a diferencia de México, fue descubierta una nueva variable denominada "consentimiento contingente". Es así como en Buenos Aires y Mar del Plata el apego a la ley depende de las características contingentes asociadas a los factores económicos, sociales y políticos, no existiendo un respeto irrestricto a la ley como en Baja California. Una explicación otorgada por los autores se relaciona con las diferencias en la consolidación democrática de ambos países. Mientras en Argentina la consolidación democrática se produjo luego de un régimen militar abusivo que imponía sus propias leyes y normas, en México la consolidación se produjo a partir de la existencia de un marco legal que era quebrantado por las autoridades políticas. Según esto, es posible plantear que en Argentina las leyes eran por tanto interpretadas como "malas leyes", mientras que en México el marco legal era validado y no así sus autoridades políticas.

Respecto a la variable "participación cívica", fue posible establecer que las regiones más democráticas tanto de Argentina como de México no fueron lugares densos en capital social, existiendo incluso el fenómeno contrario. Por otro lado, tampoco fue posible comprobar el planteamiento de la teoría del capital social respecto a que las perso-

14. El clientelismo es considerado como una estrategia partidaria cuya elección por parte de los representantes políticos estaría determinada por las creencias de los ciudadanos de cada región en torno a sus figuras políticas. 
nas que pertenecen a asociaciones o trabajan en conjunto para resolver sus problemas tienden a apoyar en mayor grado las medidas del gobierno, no existiendo una correlación estadística significativa entre ambos factores.

IV.

Como ya fue consignado, la explicación otorgada a las causas de la variación regional en la consolidación democrática constituye el aporte más destacable de esta investigación. Una vez descartada la teoría del capital social, los autores proponen un modelo explicativo que identifica la variable regional o geográfica como causante directa de las diferencias detectadas y como variables indirectas el desarrollo económico y la igualdad en el ingreso de los habitantes.

Tanto en Argentina como en México, los estados y provincias con mayor consolidación democrática corresponden a zonas industrializadas que evidencian un mayor desarrollo económico. La situación descrita podría coincidir con el planteamiento clásico que identifica el desarrollo económico y la igualdad en el ingreso como factores que influyen en el establecimiento de la democracia y en su posterior estabilidad. No obstante, esta investigación posee el mérito de comprobar que en realidad estas variables no causan de manera directa una democratización en las creencias políticas de las personas, sino que cumplen un rol indirecto a través del efecto que producen sobre el clientelismo. Se establece entonces que el desarrollo económico socava o desincentiva las prácticas clientelares al requerir de una inversión más costosa por parte de los políticos en sectores que gozan de un mayor bienestar económico y de mayor igualdad en el ingreso. Además de este argumento, los resultados de la investigación permiten desechar la variable económica como causante directa de la consolidación democrática: al analizar el caso argentino, donde las personas con ingresos más altos tienden a establecer relaciones sustentadas en la confianza personal con figuras políticas por su frecuente contacto con éstas, se descarta la relación causal que predice que a mayor riqueza personal, mayor confianza institucional. Según estos argumentos, se plantea que el desarrollo económico, así como la igualdad en el ingreso, sólo modificarían la cultura política una vez que la democracia ya se ha establecido en las regiones estudiadas (p. 164).

La variable regional se refiere al impacto que posee el lugar geográfico sobre el grado de consolidación democrática. Esta es explicada a través de dos causas: los patrones de interacción entre la élite política regional y sus votantes, y una dinámica de mutua persuasión. Las estrategias seleccionadas por la élite política estarían definidas geográficamente, es decir, los líderes políticos de determinada región optarán por una estrategia orientada 
hacia la responsabilidad política o el clientelismo, dependiendo de las creencias que posean previamente los ciudadanos ${ }^{15}$. Dicha elección a su vez refuerza la creencia existente entre los ciudadanos asentándose de esta manera un sistema de creencias consolidado, que permite que la estrategia seleccionada no genere disonancia. ¿Cómo explicar entonces el paso en una misma región desde una cultura política sustentada en la confianza personal a una sustentada en la confianza institucional? Para responder esta pregunta, se plantea el factor de la dinámica de la mutua persuasión. Éste se refiere a la influencia producida por la proximidad geográfica, otorgando la posibilidad de enviar y estar en contacto con mensajes que pueden reforzar o modificar las creencias políticas de los ciudadanos. Pese a que la proximidad geográfica puede ser delimitada al contexto de los contactos directos que poseen los ciudadanos, al interior de una misma región, reforzándose en este caso las creencias ya existentes, también debe ser extendida al campo de interacciones entre diversas regiones. Este último aspecto es gravitante al momento de explicar las modificaciones de las creencias de los ciudadanos en relación con la variable "confianza personal/institucional", señalándose que éstas son el resultado del intercambio de mensajes e información a través de las interacciones frecuentes con ciudadanos de otras regiones, así como de la exposición a mensajes transmitidos en los medios de comunicación de masas.

Una vez expuesto el modelo explicativo de esta investigación, es necesario realizar ciertas precisiones. Indudablemente, la originalidad y aporte de ésta radica en su estudio comparado regional, pudiendo develar aspectos que a nivel de comparación entre países son más difíciles de pesquisar. De esta manera, es posible establecer con claridad que las variables "desarrollo económico" e "igualdad en el ingreso" no son causas directas de una cultura política sustentada ya sea en la confianza personal o institucional. Aunque la variable geográfica funciona correctamente como explicación de la variación regional en la consolidación democrática, la explicación otorgada por los autores parece en ocasiones insuficiente, centrándose más bien en una descripción del fenómeno observado y en una interpretación de éste a través de las herramientas que brinda la teoría de juegos. Por ningún motivo esta observación implica relegar la importancia del factor geográfico en la investigación, sino subrayar que tal vez por la centralidad de éste en el resultado de su estudio, se pudo haber otorgado mayor énfasis en su explicación. Si bien es posible comprender adecuadamente por qué los ciudadanos en determinadas regiones mantienen una cultura política

15. Esta explicación es sustentada en la teoría de juegos. La elección de la estrategia política por parte de la élite regional implicaría la consideración de la cultura política regional, en tanto permite obtener mayores ganancias para éstos, al reforzar las creencias ya existentes. 
propensa más a la confianza personal o institucional, no queda del todo claro cómo ese sistema de creencias llegó a consolidarse en determinadas regiones.

Una última observación a realizar se relaciona con el concepto de cultura política que es utilizado en esta investigación, pudiendo su definición resultar insuficiente y en ocasiones restringida ${ }^{16}$. La cultura política se define solamente a través de la variable "confianza" y tomando como punto de referencia la dimensión confianza personal/institucional. Es tal vez por este motivo que el lector, al finalizar el libro, queda con una sensación de dificultad, al momento de querer asir y significar en toda su complejidad el concepto de cultura política.

16. En la parte IV del libro (capítulo 6), la cultura política es definida como patrones de acción política en relación a creencias y preferencias políticas que tienden a mostrar tanto una coherencia lógica como una covariación empírica (p. 183). 
$\perp^{\prime}$

$1 L$

$\overline{7}$

$\mid \overline{\bar{F}}$ 